\title{
Evaluación de dimetilacetamida como crioprotector para la crioconservación de semen de bocachico Prochilodus magdalenae ${ }^{\#}$
}

\author{
Evaluation of dimethylacetamide as cryoprotectant for cryopreservation \\ of sperm of bocachico Prochilodus magdalenae
}

\author{
VJ Atencio ${ }^{a^{*}}$, EJ Perez ${ }^{\mathrm{a}}$, JA Espinosa ${ }^{\mathrm{a}}$, SC Pardo \\ ${ }^{a}$ Centro de Investigación Piscícola CINPIC, Departamento de Ciencias Acuícolas, Facultad de Medicina Veterinaria \\ y Zootecnia, Universidad de Córdoba, Colombia. \\ ${ }^{\mathrm{b}}$ Grupo de Investigación Biodiversidad y Genética Molecular BIOGEM, Departamento de Producción Animal, \\ Facultad de Ciencias Agrarias, Universidad Nacional de Colombia, Sede Medellín, Medellín, Colombia.
}

\begin{abstract}
SUMMARY
The aim of this study was to e valuate the performance of dimethylacetamide (DMA) as cryoprotectant at three concentrations: $8 \%$ ( $0.85 \mathrm{M}), 10 \%$ $(1.07 \mathrm{M})$ and $12 \%(1.29 \mathrm{M})$, combined with glucose at $6 \%(0.33 \mathrm{M})$ and egg yolk at $12 \%$, to cryopreserve semen of bocachico Prochilodus magdalenae. The semen was diluted at 1:4 ratio in the cryoprotective solution, packed in $2.5 \mathrm{~mL}$ straws and frozen in liquid nitrogen vapor $\left(\mathrm{LN}_{2}\right)$ for 30 minutes, then it was stored in a container with $\mathrm{LN}_{2}$ of $34 \mathrm{~L}$. The concentration, total motility, progressive motility and sperm speed in both fresh (control) and cryopreserved-thawed semen were evaluated using Sperm Class Analyzer (SCA). Straws were thawed in serological bath at $60^{\circ} \mathrm{C}$ for 45 seconds. Fertility and hatching were evaluated inseminating $2 \mathrm{~g}$ of eggs $(1,540 \mathrm{eggs} / \mathrm{g})$ at a rate of 320,000 spz/egg. Fresh semen recorded the highest values of total motility, total progressive, curvilinear speed and linear speed with regard to cryopreserved-thawed semen $(\mathrm{P}<0.05)$; whereas cryopreservedthawed semen with DMA $8 \%$ and $10 \%$ recorded the higher values for these variables, without significant differences between them (P > 0.05). DMA $12 \%$ reported the lowest values of all the variables evaluating semen quality. Fresh semen obtained values of $74.0 \pm 5.3 \%$ and $62.3 \pm 3.1 \%$ for fertility and hatching, respectively, while cryopreserved-thawed semen with DMA at $8 \%$ produced adequate fertility $(60.4 \pm 8.4 \%)$ and hatching $(50.1 \pm 9.8 \%)$ values. The results of this study suggest that the cryoprotectant solution composed of DMA at $8 \%$, glucose at $6 \%$ and egg yolk at $12 \%$ is a viable alternative for the cryopreservation of semen of bocachico.
\end{abstract}

Key words: dimethylacetamide, cryopreservation, milt, Prochilodus magdalenae.

\section{RESUMEN}

Se evaluó dimetilacetamida (DMA) como crioprotector, a tres concentraciones: $8 \%(0,85 \mathrm{M}), 10 \%(1,07 \mathrm{M})$ y $12 \%(1,29 \mathrm{M})$ combinado con glucosa $6 \%(0.33 \mathrm{M})$ y yema de huevo $12 \%$ para crioconservar semen de bocachico Prochilodus magdalenae. El semen fue diluido en proporción 1:4 en la solución crioprotectora, empacado en pajuelas de $2,5 \mathrm{~mL}$ y congelado en vapores de nitrógeno líquido $\left(\mathrm{N}_{2} \mathrm{~L}\right)$ durante 30 minutos y luego almacenados en un termo de $\mathrm{N}_{2} \mathrm{~L}$ de $34 \mathrm{~L}$. La concentración, movilidad total, progresividad y velocidad tanto en semen fresco (control) como en semen crioconservadodescongelado se evaluó con ayuda de Sperm Class Analyzer (SCA). Las pajuelas fueron descongeladas en baño serológico a $60^{\circ} \mathrm{C}$ durante 45 segundos. La fertilidad y eclosión fueron evaluadas inseminando dos gramos de huevos (1540 huevos/g) a razón de 320.000 spz/ovocito. El semen fresco registró mayores valores de movilidad total, progresividad total, velocidad curvilínea y velocidad lineal con respecto al semen crioconservado-descongelado $(\mathrm{P}<0,05)$; mientras que el semen crioconservado-descongelado con DMA 8\% y 10\% registraron los mayores valores en estas mismas variables, sin observarse diferencia significativa entre ellos $(\mathrm{P}>0,05)$. DMA $12 \%$ reportó los menores valores de todas las variables que evaluaron la calidad seminal. Con semen fresco se obtuvo fertilidad de 74,0 $\pm 5,3 \%$ y eclosión de $62,3 \pm 3,1 \%$; mientras que con semen crioconservado-descongelado con DMA $8 \%$ se obtuvo adecuada fertilidad $(60,4 \pm 8,4 \%)$ y eclosión $(50,1 \pm 9,8 \%)$. Los resultados del estudio sugieren que la solución crioprotectora compuesta por DMA $8 \%$, glucosa $6 \%$ y yema de huevo $12 \%$ es una alternativa viable para la crioconservación de semen de bocachico.

Palabras clave: dimetilacetamida, crioconservación, semen, Prochilodus magdalenae.

\section{INTRODUCCIÓN}

A pesar de que el bocachico Prochilodus magdalenae es la principal especie de pesquería continental colombia-

Aceptado: 29.11.2013.

\# Estudio financiado por el Ministerio de Agricultura y Desarrollo Rural, Colombia.

* Cra 6 Nº 76-103, Montería, Córdoba, Colomba; vatencio@hotmail.com na, ésta ha sido declarada en peligro de extinción (Mojica y col 2002); debido a que sus volúmenes de capturas en los últimos cuarenta años, en la cuenca del río Magdalena, han disminuido drásticamente pasando de 60.721 toneladas promedio/año en la década del 70 a 6.075 toneladas promedio/año en la década pasada (2000-2009) (CCI 2010). También es una especie con importancia en la piscicultura rural colombiana, alcanzando valores de 2.545 toneladas en 2005 (CCI 2009); que la ubican como 
la cuarta especie íctica más cultivada en Colombia después de tilapia (Oreochromis sp, Oreochromis niloticus), cachama (Piaractus brachypomus, Colossoma macropomum) y trucha (Onchorynchus mykiss) y la segunda nativa después de la cachama. La demanda de alevines de bocachico se ha incrementado en virtud de la expansión de su cultivo y la ejecución de programas de repoblamiento en las principales cuencas hidrográficas del país (Caraballo y col 2003, Atencio y col 2010), por lo que el desarrollo piscícola requiere de la adopción de tecnologías como la crioconservación de semen para apoyar la optimización de los sistemas de producción actuales. Los beneficios de la crioconservación de semen son ampliamente conocidos e incluyen desde facilitar el mejoramiento genético de las especies de interés comercial hasta permitir la conservación de especies amenazadas o en peligro de extinción (Wildt y Wemmer 1999, Watson y Holt 2001, Medina-Robles y col 2005). La crioconservación de semen de peces en nitrógeno líquido ha sido reportado en más de 83 especies de peces, particularmente salmónidos, ciprínidos y bagres con importancia comercial, permitiendo un mayor control en la reproducción (Tiersch 2011). La colección del semen, composición del diluyente, concentración del crioprotector, curvas de congelación y descongelación, entre otras, son variables importantes en el éxito de los protocolos de crioconservación de semen (Tiersch 2011, Irawan y col 2010).

En Colombia, los estudios de crioconservación de semen de peces nativos son recientes y se han orientado principalmente a la estandarización de protocolos de congelación, evaluación de crioprotectores y diluyentes para disminuir los efectos tóxicos y el criodaño sobre la célula espermática (Medina-Robles y col 2005, Martínez 2010, Martínez y col 2011). Se destacan los avances en el desarrollo de protocolos de crioconservación de yamú Brycon amazonicus (Cruz-Casallas y col 2006, Velasco-Santamaría y col 2006), cachama blanca Piaractus brachypomus (Navarro y col 2004, Ramírez-Merlano y col 2005) y bagre rayado Pseudoplatystoma metaense (Ramírez-Merlano y col 2011) con resultados satisfactorios en la reproducción artificial de estas especies con semen crioconservado.

Los diluyentes y crioprotectores tienen como función incrementar el volumen del eyaculado, proteger al espermatozoide de la acción tóxica de los productos del metabolismo celular y de los cambios bruscos de temperatura (Medina-Robles y col 2005). Los crioprotectores deben presentar baja toxicidad para la célula y alta solubilidad en el agua y, dependiendo de su capacidad de permear la membrana celular, se les clasifica en intracelulares (permeables) o extracelulares (no permeables) (Denniston y col 2011). Dimetilsulfóxido (DMSO) es uno de los crioprotectores intracelulares de bajo peso molecular, muy común en la crioconservación de semen de teleósteos y en particular de Characiformes del neotrópico, reportándose su utilización en Prochilodus scrofa, Salminus brasiliensis (Coser y col 1984, Viveiros y col 2009a),
Leporinus elongatus (Carolsfeld y col 2003), Piaractus mesopotamicus (Fogli da Silveira y col 1990, Carolsfeld y col 2003), cachama blanca (Navarro y col 2004, Nascimento y col 2010), yamú (Cruz-Casallas y col 2006), piracanjuba Brycon orbignyanus (Carolsfeld y col 2003), Prochilodus lineatus (Viveiros y col 2009 ${ }^{\mathrm{b}}$ ), bocachico (Martínez 2010, Arroyo y Vergara 2010); sin embargo, dimetilacetamida (DMA), otro crioprotector de bajo peso molecular y de acción intracelular, ha sido poco evaluado en Characiformes, pero con buenos resultados en la crioconservación de semen de Siluriformes como en Clarias gariepinus (Urbanyí y col 1999), Silurus glanis (Ogier María de Baulny y col 1999) y en Pseudoplatystoma metaense (Ramírez-Merlano y col 2011), también ha mostrado buenos resultados en trucha arco iris Oncorhynchus mykiss (McNiven y col 1993, Babiak y col 2001) y carpa común Cyprinus carpio (Warnecke y Pluta 2003).

La yema de huevo es un crioprotector no permeable (extracelular) que tiene una acción termoprotectora, ejercida por la fracción lipídica compuesta por lecitina y cefalina y una acción conservadora dada por la fracción lipoproteíca de la yema de huevo (María y col 2006); lo cual la ha convertido en uno de los crioprotectores no permeables más utilizados en semen de mamíferos y peces de agua dulce por su capacidad de prevenir daños a la membrana. Babiak y col (2001) encontraron un efecto benéfico en la combinación de DMA como crioprotector intracelular y yema huevo como crioprotector extracelular, cuando crioconservaron semen de trucha arco iris. María y col (2006) observaron que en piracanjuba la movilidad del semen post-descongelación estuvo fuertemente correlacionada con el uso de la yema de huevo en la solución crioprotectora. Los diluyentes utilizados en peces han sido formulados simulando la composición y osmolaridad del plasma seminal de cada especie para mantener inactivos los espermatozoides cuando es diluido antes de la congelación debido a la estabilización de las propiedades fisicoquímicas (Ohta y Izawa 1996, Medina-Robles y col 2005). En bocachico, Martínez y col (2011) encontraron que la osmolaridad seminal de bocachico oscila entre 250 y $300 \mathrm{mOsm} / \mathrm{Kg}$ y sugirieron que la glucosa a concentración de $6 \%(360 \mathrm{mOsm} / \mathrm{Kg})$ es un diluyente no activador de la movilidad espermática.

El objetivo del presente estudio fue evaluar DMA como crioprotector en la crioconservación de semen de bocachico Prochilodus magdalenae con el fin de favorecer la disponibilidad de semen durante todo el año y contribuir con los programas productivos y de conservación de esta especie.

\section{MATERIAL Y MÉTODOS}

\section{MANEJO DE REPRODUCTORES Y RECOLECCIÓN DE SEMEN}

El estudio se realizó en las instalaciones del Centro de Investigación Piscícola de la Universidad de Córdo- 
ba (CINPIC). Se utilizaron machos $(n=12)$ y hembras $(\mathrm{n}=4)$ adultos de bocachico, de dos a tres años de edad, mantenidos bajo condiciones de cautiverio en estanques en tierra, a densidad de $0,5 \mathrm{Kg} / \mathrm{m}^{2}$, con recambio de agua de $15 \%$ semanal. Los machos, con peso promedio de $0,169 \pm 0,024 \mathrm{Kg}$ y longitud total promedio de $25,2 \pm$ $1,8 \mathrm{~cm}$, se seleccionaron en época de espermiación, es decir cuando mediante leve presión sobre la cavidad celómica en sentido cráneo-caudal liberaron líquido seminal; mientras que las hembras, con peso promedio de 0,226 \pm $0,063 \mathrm{Kg}$ y longitud total promedio de $29,5 \pm 0,8 \mathrm{~cm}$, se seleccionaron durante la época de maduración final del ovocito, lo cual fue verificado observando la posición de la vesícula germinal en una biopsia ovárica (Atencio 2001). Todos los ejemplares fueron trasladados a tanques rectangulares de fibra de vidrio de $2,4 \mathrm{~m}^{3}$ de capacidad, con flujo continuo de agua $(6 \mathrm{~L} / \mathrm{min})$ donde permanecieron durante $48 \mathrm{~h}$, con el propósito de adaptarlos a las condiciones experimentales, reducir el estrés generado por la manipulación y cambio de ambiente. Posteriormente se administró extracto pituitario de carpa (CPE, Argent Chem Lab, Redmond, WA, EUA) para inducir la ovulación en las hembras e incrementar el volumen seminal en los machos. A las hembras se les administró $5 \mathrm{mg}$ de $\mathrm{CPE} / \mathrm{Kg}$ de peso, en dos inyecciones, una inicial equivalente al $20 \%$ y doce horas después el $80 \%$ restante; mientras que los machos fueron tratados con $4 \mathrm{mg}$ de $\mathrm{CPE} / \mathrm{Kg}$ en dosis única (Atencio 2001).

Antes de la recolección del semen, la papila urogenital se secó con papel absorbente. El semen se colectó después de siete horas de aplicada la sustancia inductora (CPE), mediante masaje abdominal en sentido cráneocaudal colectándolo directamente en tubos Eppendorf graduados de $2 \mathrm{~mL}$, estériles y secos. Se registró el color para evidenciar la posible presencia de sustancias contaminantes, considerándose blanco el color de una muestra no contaminada. Muestras contaminadas con heces u orina fueron descartadas. Una hora después de colectar el semen de los machos, fueron colectados los desoves de las hembras por masajes abdominales y recibidos en recipientes plásticos.

\section{EVALUACIÓN DE LA CALIDAD SEMINAL}

Para evaluar la movilidad se utilizó una muestra de $0,25 \mu \mathrm{l}$ de semen y $75 \mu \mathrm{l}$ de agua bidestilada (dilución 1:300) en una cámara de conteo Makler $(10 \mathrm{ml}$, Sefi Medical Instruments Ltd, Israel), dispuesta en un microscopio óptico de contraste de fase (Nikon, E50i, Japón) y el programa asistido por computadora para análisis de semen Sperm Class Analyzer SCA (Microptic SL, SCA VET 01, España). Solamente usadas muestras de semen con movilidad mayor de $80 \%$ fueron usados en este estudio. El tiempo de activación se determinó desde el momento en el cual se adicionó la solución activadora (agua bidestilada) a la muestra de semen, hasta que aproximadamente el $90 \%$ de los espermatozoides dejó de moverse. La velocidad espermática se analizó con ayuda del programa SCA, obteniéndose el porcentaje de espermatozoides que mostraron velocidad rápida (tipo a), caracterizados por velocidades mayores a $100 \mu \mathrm{m} / \mathrm{s}$, media (tipo b) entre 46 y $100 \mu \mathrm{m} / \mathrm{s}$ y lentos (tipo c) entre 10 y 45 $\mu \mathrm{m} / \mathrm{s}$, así como el porcentaje de espermatozoides inmóviles (tipo d). Adicionalmente, se estimó la velocidad curvilínea (VCL) y lineal (VSL). Para medir la concentración espermática se utilizó $1 \mu \mathrm{l}$ de semen mezclado con $699 \mu \mathrm{l}$ de una solución de glucosa al 6\% en un tubo Eppendorf de $2 \mathrm{~mL}$ (dilución 1:700) (Martínez 2010), la mezcla se homogenizó durante cinco segundos en un vortex a 1200 rpm (Velp Scientific, Zxclasic, China). Luego se tomó una muestra de $10 \mu \mathrm{l}$ y se colocó en la cámara Makler para la determinación de la concentración con ayuda de SCA, este procedimiento se repitió tres veces, y se calculó la concentración promedio de la muestra de semen.

\section{DISEÑO EXPERIMENTAL}

Se evaluó DMA (N,N-Dimethylacetamide, JT Baker J 372-07, Alemania) como crioprotector a tres concentraciones: $8 \%(0,85 \mathrm{M}), 10 \%(1,07 \mathrm{M})$ y $12 \%(1,27 \mathrm{M})$ para la crioconservación de semen de bocachico. La solución crioprotectora se preparó con $6 \%$ de glucosa (peso/volumen, p/v, 0,33 M), $12 \%$ yema de huevo (v/v) y DMA a las diferentes concentraciones $(\mathrm{v} / \mathrm{v})$. Se preparó una mezcla (pool) de semen mezclado en partes iguales de semen colectado de cuatro machos. El pool fue diluido en proporción 1:4 (semen: solución crioprotectora), a temperatura de $28 \pm 1^{\circ} \mathrm{C}$ y empacado en pajuelas de $5 \mathrm{~mL}$ (Minitube 13441/0280, Alemania) previamente selladas con polivinilo y esferas de acero inoxidable y congeladas en termo seco de vapores de nitrógeno líquido de $4 \mathrm{~L}$ (MVE, SC 4/2, Alemania) durante 30 minutos. La tasa de refrigeración-congelación fue de $27,3^{\circ} \mathrm{C} / \mathrm{min}$ desde 28 a $-20^{\circ} \mathrm{C}$, de $29,9^{\circ} \mathrm{C} / \mathrm{min}$ desde -20 a $-100^{\circ} \mathrm{C}$ y $5,5^{\circ} \mathrm{C} / \mathrm{min}$ desde -100 a $-196^{\circ} \mathrm{C}$. Luego las pajuelas fueron trasladadas a un termo de almacenamiento de $30 \mathrm{~L}$ (MVE, XC 34/18, Alemania) hasta el momento de su evaluación. Las pajuelas fueron descongeladas por inmersión directa en baño de agua (Memmert, WNB 7-45, Alemania) a $60^{\circ} \mathrm{C}$, durante $45 \mathrm{~s}$. Inmediatamente uno de los extremos de las pajuelas fue cortado con tijera y una muestra de semen descongelado fue evaluada (movilidad, progresividad y velocidad espermática).

\section{PRUEBAS DE FERTILIDAD Y ECLOSIÓN}

La fertilidad y eclosión se evaluaron con dos gramos de ovocitos, inseminados en una proporción de 320.000 spz/ovocito (Arroyo y Vergara 2010), para lo cual, previamente se calculó el número promedio de ovocitos por gramo (aprox. 1540 ovocito/g) y los volúmenes seminales requeridos para la cantidad de ovocitos se adicionaron 
con micropipetas (Transferpette ${ }$, CE704174, Alemania). La activación del semen en los dos gramos de ovocitos se realizó con $5 \mathrm{~mL}$ de agua destilada. El porcentaje de fertilidad se evaluó a las cinco horas post-fertilización (HPF) con una muestra de por lo menos 50 embriones tomados al azar con una pipeta de vidrio de $0,5 \mathrm{~cm}$ de diámetro y observados con un estereoscopio óptico (20X). Se consideraron huevos fertilizados aquellos de apariencia translúcida y con clara evidencia de división celular homogénea, mientras que los no fertilizados a los opacos y blanquecinos. Finalmente el porcentaje de eclosión se evaluó a las $10 \mathrm{HPF}$, de la misma manera que fue evaluada la fertilidad.

\section{ANÁLISIS ESTADÍSTICO}

Se utilizó un diseño completamente al azar. Todas las variables analizadas se transformaron mediante la función arcoseno y luego se sometieron a pruebas de normalidad (Test de Kolmogorov-Smirnov) y homogeneidad de varianzas (Test de Levene`s). Se realizó análisis de varianza (ANDEVA) y cuando se presentaron diferencias estadísticas se aplicó la prueba de comparación múltiple de Tukey. En todos los casos se utilizó un nivel de significancia de 5\%. Todos los análisis estadísticos fueron realizados en SAS versión 9.0 para Windows (SAS Institute Inc, EUA).

\section{RESULTADOS}

El semen fresco (control) de bocachico presentó color blanco, con volumen seminal promedio de 1,3 \pm $0,4 \mathrm{~mL}$, con concentración espermática de $18524,0 \times 10^{6}$ $\pm 3452,4 \times 10^{6} \mathrm{spz} / \mathrm{mL}$ y movilidad superior a $90 \%$. El semen fresco mostró los mayores valores de movilidad total, movimiento progresivo total, velocidad curvilínea y velocidad lineal, observándose diferencia significativa con los valores registrados con semen crioconservadodescongelado a las diferentes concentraciones de DMA ( $\mathrm{P}<0,05)$ (cuadro 1). El semen crioconservado-descongelado con DMA $8 \%$ y DMA $10 \%$ registraron los mayores valores de movilidad total, movilidad progresiva total, velocidad curvilínea y velocidad lineal, sin observarse diferencia significativa entre estos dos tratamientos ( $\mathrm{P}<0,05)$; mientras DMA 12\% reportólos menores valores $(\mathrm{P}<0,05)$ para estas mismas variables (cuadro 1$)$.

El semen fresco presentó el mayor porcentaje de espermatozoides de velocidad tanto rápida como media y los menores valores de espermatozoides con movilidad lenta e inmóviles, observándose diferencia significativa con los valores presentados por los espermatozoides crioconservados-descongelados con DMA $(\mathrm{P}<0,05)$ (cuadro 1). El semen crioconservado-descongelado presentó el mayor número de espermatozoides con movilidad rápida cuando se utilizó DMA 10\%, siendo estadísticamente diferente de los valores obtenidos con DMA 8 y $12 \%$ $(\mathrm{P}<0,05)$, mientras que la mayor cantidad de espermatozoides con velocidad media se registraron con DMA 8 y10\% sin registrarse diferencias significativas $(\mathrm{P}>0,05)$ entre estos valores. El porcentaje de espermatozoides lentos no registró diferencias estadísticas entre los tratamientos evaluados con semen crioconservado-descongelado con DMA $(\mathrm{P}>0,05)$ mientras que los mayores valores de espermatozoides inmóviles fueron registrados con DMA $12 \%(\mathrm{P}<0,05)$ (cuadro 1$)$.

Los mayores valores de movilidad progresiva tipo a y b se registraron con semen fresco, así como los menores valores de movilidad progresiva tipo c y d (cuadro 1),

Cuadro 1. Características de semen fresco (control) y crioconservado-descongelado de bocachico Prochilodus magdalenae. Characteristics of fresh (control) and cryopreserved-thawed semen of bocachico Prochilodus magdalenae.

\begin{tabular}{lcccc}
\hline \multicolumn{1}{c}{ Características } & Semen fresco & DMA $8 \%$ & DMA 10\% & DMA 12\% \\
\hline Movilidad total $(\%)$ & $98,8 \pm 1,1^{\mathrm{a}}$ & $31,0 \pm 8,6^{\mathrm{b}}$ & $30,9 \pm 1,0^{\mathrm{b}}$ & $18,7 \pm 0,7^{\mathrm{c}}$ \\
Movilidad rápida $(\%)$ & $85,5 \pm 1,0^{\mathrm{a}}$ & $6,9 \pm 0,8^{\mathrm{c}}$ & $8,6 \pm 1,0^{\mathrm{b}}$ & $0,7 \pm 0,4^{\mathrm{d}}$ \\
Movilidad media $(\%)$ & $7,3 \pm 1,0^{\mathrm{a}}$ & $5,4 \pm 1,4^{\mathrm{b}}$ & $3,8 \pm 0,2^{\mathrm{b}}$ & $0,8 \pm 0,2^{\mathrm{c}}$ \\
Movilidad lenta $(\%)$ & $6,3 \pm 0,3^{\mathrm{b}}$ & $18,8 \pm 8,5^{\mathrm{a}}$ & $20,9 \pm 4,3^{\mathrm{a}}$ & $17,5 \pm 0,7^{\mathrm{a}}$ \\
Inmóviles $(\%)$ & $1,1 \pm 0,4^{\mathrm{c}}$ & $69,1 \pm 8,6^{\mathrm{b}}$ & $66,8 \pm 3,2^{\mathrm{b}}$ & $81,1 \pm 0,8^{\mathrm{a}}$ \\
Velocidad curvilínea $(\mu \mathrm{m} / \mathrm{seg})$ & $186,6 \pm 15,4^{\mathrm{a}}$ & $63,2 \pm 11,6^{\mathrm{b}}$ & $54,7 \pm 0,9^{\mathrm{b}}$ & $21,8 \pm 4,0^{\mathrm{c}}$ \\
Velocidad lineal $(\mu \mathrm{m} / \mathrm{seg})$ & $87,4 \pm 14,7^{\mathrm{a}}$ & $36,4 \pm 5,0^{\mathrm{b}}$ & $26,5 \pm 1,3^{\mathrm{b}}$ & $6,0 \pm 0,5^{\mathrm{c}}$ \\
Progresividad total $(\%)$ & $79,1 \pm 12,3^{\mathrm{a}}$ & $11,0 \pm 2,6^{\mathrm{b}}$ & $10,7 \pm 0,8^{\mathrm{b}}$ & $1,4 \pm 0,2^{\mathrm{c}}$ \\
Progresividad tipo a (\%) & $26,4 \pm 1,2^{\mathrm{a}}$ & $2,9 \pm 1,0^{\mathrm{b}}$ & $2,0 \pm 0,1^{\mathrm{b}}$ & $0,0 \pm 0,1^{\mathrm{c}}$ \\
Progresividad tipo b (\%) & $61,5 \pm 10,4^{\mathrm{a}}$ & $8,1 \pm 2,8^{\mathrm{b}}$ & $8,7 \pm 0,8^{\mathrm{b}}$ & $1,1 \pm 0,4^{\mathrm{b}}$ \\
Progresividad tipo c $(\%)$ & $10,9 \pm 1,6^{\mathrm{c}}$ & $19,1 \pm 1,0^{\mathrm{b}}$ & $22,5 \pm 3,0^{\mathrm{a}}$ & $17,8 \pm 1,1^{\mathrm{b}}$ \\
Progresividad tipo d $(\%)$ & $1,2 \pm 0,4^{\mathrm{b}}$ & $69,1 \pm 7,4^{\mathrm{a}}$ & $66,8 \pm 12,1^{\mathrm{a}}$ & $81,1 \pm 6,6^{\mathrm{a}}$ \\
\hline
\end{tabular}

Letras diferentes en la misma fila indican diferencia estadística $(\mathrm{P}<0,05)$. 
observándose diferencia significativa $(\mathrm{P}<0,05)$ con los tratamientos evaluados con las diferentes concentraciones de DMA. Con semen crioconservado-descongelado los mayores valores de movilidad progresiva tipo a se obtuvieron con DMA 8 y 10\% (cuadro1), sin registrarse diferencia estadística $(\mathrm{P}>0,05)$ entre estos valores, mientras que los valores de movilidad progresiva tipo $b$ no registraron diferencia significativa $(P>0,05)$ entre el semen descongelado. El mayor porcentaje de movilidad progresiva tipo c se obtuvo con DMA $10 \%(\mathrm{P}<0,05)$ y la movilidad progresiva tipo d (cuadro 1) no registró diferencia significativa $(\mathrm{P}>0,05)$ entre los tratamientos con semen descongelado.

El semen fresco registró la mayor tasa de fertilidad de los tratamientos evaluados $(74,0 \pm 5,3 \%)$; y en los tratamientos evaluados con semen crioconservado-descongelado el mayor porcentaje de fertilidad se observó cuando se utilizó DMA $8 \%(60,4 \pm 8,4 \%)$ y la menor con DMA $12 \%(22,4 \pm 7,1 \%)$ (figura 1), observándose diferencia significativa entre estos tratamientos $(\mathrm{P}<0,05)$. También el semen fresco registró la mayor tasa de eclosión (62,3 \pm $3,1 \%$ ), mientras que con semen crioconservado-descongelado la mayor tasa de eclosión se obtuvo con DMA $8 \%(50,1 \pm 9,8 \%)$ y la menor cuando se utilizó de DMA $12 \%(11,2 \pm 6,7 \%)$, observándose diferencia significativa entre estos valores $(\mathrm{P}<0,05)$ (figura 1$)$.

\section{DISCUSIÓN}

Las variables más utilizadas para medir la calidad espermática son movilidad, velocidad y progresividad (Moore y Akhondi 1996). La movilidad total del semen fresco fue tres veces superior a la mayor movilidad observada en el semen crioconservado-descongelado (DMA $8 \%$ ); sin embargo, Arroyo y Vergara (2010) observaron altos porcentajes de movilidad total $(76,8 \pm 5,9 \%)$ con semen de bocachico crioconservado con DMSO 10\%, aproximadamente dos veces la obtenida en el presente estudio con DMA 8 y 10\%. Martínez y Pardo (2010, en una reciente revisión sobre los efectos de la crioconservación sobre la calidad y el desempeño espermático, encontraron que hasta el momento esto se debe a daños en el genoma y en el proteoma. Los daños en el genoma impiden la replicación y la transcripción de genes mitocondriales o nucleares lo que a su vez impide la síntesis de proteínas claves en la producción energética afectando la movilidad; los daños en el proteoma se traducen en la interrupción de la cascada de fosforilación y activación enzimática, lo que afecta la movilidad del espermatozoide, así como su capacidad fertilizante. Ogier de Baulny y col (1999) señalaron que la crioconservación de semen ocasiona daños a estructuras celulares como membrana, núcleo, mitocondria y flagelo, los cuales afectan la calidad

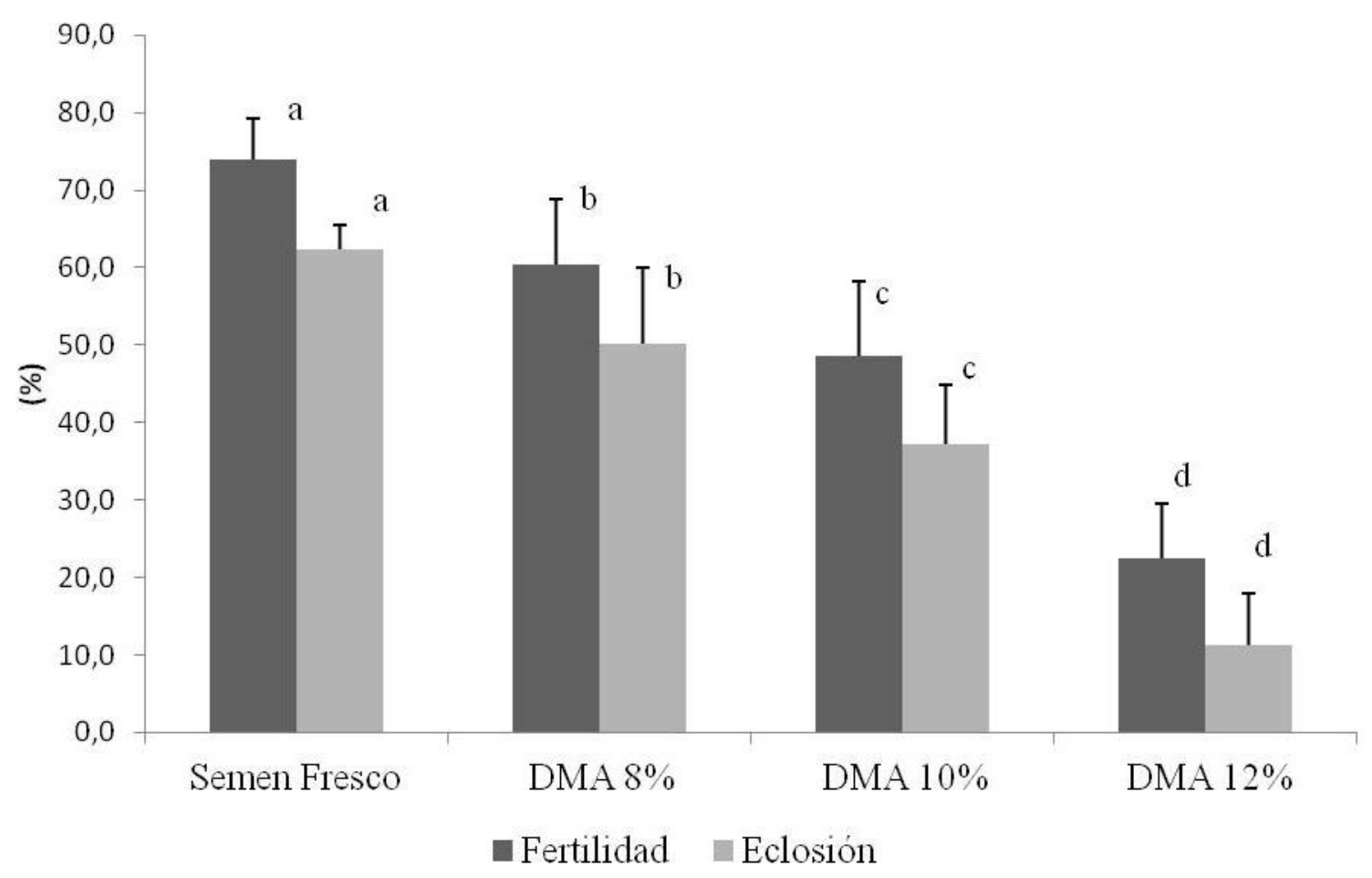

Figura 1. Tasas de fertilidad y de eclosión de bocachico Prochilodus magdalenae inseminado con semen fresco y crionservado-descongelado con DMA a diferentes concentraciones. Entre tratamientos, valores con letras distintas son significativamente diferentes $(\mathrm{P}<0,05)$.

Fertility and hatching rates of bocachico Prochilodus magdalenae fertilized with fresh and cryopreserved-thawed semen with DMA at different concentrations. Between bars, values with different letters are significantly different $(\mathrm{P}<0.05)$. 
del espermatozoide. Según Kurland y Andersson (2000) la pérdida de movilidad en el semen post-descongelado puede estar asociada con daños en la mitocondria, directamente sobre su ADN o su membrana, o de manera indirecta por la fragmentación del ADN nuclear necesario para la obtención de proteínas no codificadoras del genoma por parte de la mitocondria. Ogier de Baulny y col (1999) reportaron alta movilidad total, cercana a 60\%, cuando utilizaron DMA 10 y $15 \%$ en la crioconservación de semen de Silurus glanis, por encima de la obtenida con DMSO y metanol. Richardson y col (2000) reportaron movilidad total 31,7\% con semen post-descongelado de Salvelinus alpinus crioconservado con DMA 10\%, similar a la obtenida en el presente estudio con DMA 8 y $10 \%$. Sin embargo Gwo y col (1999) reportaron una baja movilidad total de semen post-descongelado crioconservado con DMA para Onchorynchus masou formasanus.

Según Martínez y col (2012) la calidad seminal no sólo debería evaluarse estimando la movilidad o fertilidad sino que también deberían utilizarse variables que expliquen el grado genotoxicidad y citotoxicidad de los crioprotectores, tales como fragmentación DNA y daños en la membrana, los cuales podrían revelar con mayor precisión la esencia y el origen de los problemas que no son observados mediante las variables macroscópicas.

Según Martínez (2010) daños a la membrana provocados por la crioconservación podrían tener efectos sobre la movilidad y fecundidad, entre otros; haciendo inviable al espermatozoide; sin embargo este mismo autor señala que el proceso de difusión lateral de moléculas (lípidos y proteínas), podría permitir la estabilización transitoria de la membrana plasmática durante el proceso de crioconservación; en el cual la distribución homogénea de partículas y la relación de fosfolípidos y proteínas hacen posible la reversión exitosa de la célula espermática para conservar su capacidad fecundante; fenómeno que podría explicar la fecundación con bajos porcentajes de movilidad.

Martínez y col (2012) evaluaron diferentes protocolos de crioconservación de semen de bocachico con DMSO $(5,10$ y $15 \%)$, glucosa $(5,5,5$ y $6 \%)$ y yema de huevo $12 \%$; encontraron que la crioconservación produce daños significativos en la fragmentación de DNA y daños en membrana cuya magnitud depende de la concentración del diluyente (glucosa) y del crioprotector (DMSO) y sus interacciones; además sugirieron que los daños son menores cuando se usa DMSO a $10 \%$ en combinación con glucosa $(5,5$ o $6 \%)$.

En este estudio, en semen fresco, los espermatozoides de movilidad rápida más los de media sumaron más del $90 \%$ y los inmóviles no superaron $2 \%$, mientras que en semen descongelado la suma de rápidos y medios no alcanzaron $13 \%$, predominando, en los tratamientos de DMA, los inmóviles $(69,1-81,8 \%)$. A pesar de los bajos porcentajes de espermatozoides rápidos, medios y lentos, se obtuvo buena fertilidad con DMA 8 y $10 \%$. Arroyo y Vergara (2010) obtuvieron 37,8\% de espermatozoides rápidos más medios con semen de bocachico crioconservado con DMSO 10\%, obteniendo fertilidad cercana a $70 \%$, similar a la obtenida en el presente estudio.

Los mayores valores de velocidad lineal (VSL) y curvilínea (VCL) se obtuvieron con semen fresco, mientras que con crioconservado-descongelado los mayores valores de VSL y VCL se obtuvieron con DMA 8\% y $10 \%$, pero con reducción de la velocidad a la mitad o tercera parte de la observada en el semen fresco. Estas velocidades están ligeramente por encima de las reportadas por Arroyo y Vergara (2010) con semen de bocachico crioconservado con DMSO 10\% (VCL $=49,9 \pm$ $7,7 \mu \mathrm{m} / \mathrm{seg}$ y VSL $=33,6 \pm 7,8 \mu \mathrm{m} / \mathrm{seg}$ ). Según Li y col (2008) las pérdidas de velocidad VCL y VSL ocurridas luego de los procesos de congelación y descongelación son causadas por daños en la integridad del ADN, hecho que es posible cuando la osmolaridad y la concentración de los crioprotectores no son apropiadas. Los registros de velocidades espermáticas post-descongelación de semen de Characiformes crioconservado con DMA son escasos; pero resultados en otros grupos de peces, como los obtenidos por Rurangwa y col (2001) quienes utilizaron DMSO $10 \%$ y reportaron VCL $(58 \mu \mathrm{m} / \mathrm{seg})$ y VSL (38 $\mu \mathrm{m} / \mathrm{s}$ ) similares a los obtenidos en el presente estudio con DMA 8 y 10\%; mientras que Lahnsteiner y col (1996) reportaron valores mayores de VCL $(87,7 \mu \mathrm{m} / \mathrm{s})$ y VSL $(86,2 \mu \mathrm{m} / \mathrm{s})$ para Oncorhynchus mykiss con DMSO $10 \%$.

El movimiento progresivo total se redujo aproximadamente a la séptima parte en semen descongelado crioconservado con DMA 8 y $10 \%$ cuando es comparada con la obtenida en semen fresco. Arroyo y Vergara (2010) reportaron progresividades total de $29,0 \pm 4.5 \%$ con DMSO $10 \%$, así como las progresividad tipos a $(6,0$ $\pm 1,8 \%)$ y b $(23,1 \pm 2,8 \%)$, las cuales resultaron entre dos y tres veces las obtenidas en el presente estudio.

La inseminación con semen fresco registró las mayores tasas de fertilidad $(70,0 \pm 5,3 \%)$ y eclosión $(62,3 \pm$ $3,1 \%$ ); mientras que con semen crioconservado a las diferentes concentraciones de DMA evaluadas ( 8,10 y $12 \%)$ fueron decreciendo a medida que aumentó la concentración del crioprotector. La mayor fertilidad con semen crioconservado-descongelado se obtuvo cuando se utilizó la menor concentración de DMA $8 \%(60,4 \pm 8,4 \%)$, mientras que la menor se obtuvo con DMA $12 \%(22,4 \pm$ $7,1 \%$ ). A pesar de las bajas movilidades obtenidas con DMA $8 \%$ (menores de $40 \%$ ) los porcentajes de fertilidad pueden ser considerados buenos $(60,4 \%)$. Esta tendencia de bajas movilidades y altas fertilidades también fueron reportadas por Ramírez-Merlano y col (2011) quienes registraron movilidades inferiores a $40 \%$ en Pseudoplatystoma metaense con fertilidades superiores a $60 \%$. Navarro y col (2004) con semen de Piaractus brachypomus utilizaron etilenglicol 5\% y registraron movilidades de $37 \%$ con fertilidad de $38 \%$ y con propilenglicol $5 \%$ registraron las mayores movilidades (64\%) con fertilidades 
casi nulas (2\%). Martínez (2010) afirmó que la fertilidad exige del espermatozoide más que una buena movilidad o una variable en particular, el bienestar integral del espermatozoide o por lo menos en la mayor parte de sus variables. El mismo comportamiento se observó con la tasa de eclosión. Estos resultados sugieren que concentraciones de 10 y $12 \%$ de DMA pueden ser más tóxicas para el espermatozoide de bocachico; causándole una disminución de su calidad y por ende afectando su capacidad fertilizante. Cruz-Casallas y col (2006) evaluando DMSO como crioprotector, para la crioconservación de espermatozoides de Brycon amazonicus, observaron el mismo comportamiento, y señalaron que mayores concentraciones de los crioprotectores resultan más tóxicos para las células espermáticas. Por tal razón, Chao (1991) sugirió, considerando los efectos tóxicos de los crioprotectores y diluyentes sobre los espermatozoides, la necesidad de determinar para cada especie tanto el crioprotector como el diluyente más adecuado, así como la concentración que se deben utilizar. Los resultados de fertilidad y eclosión de semen de bocachico crioconservado con DMA $8 \%$, permiten sugerir que este crioprotector es una alternativa para la crioconservación de semen de esta especie; ya que cuando se le compara con los resultados obtenidos por Arroyo y Vergara (2010) utilizando DMSO 10\% en la crioconservación de semen de bocachico, usando dosis inseminante de 320.000 spz/ovocito, obtuvieron fertilidad $(70,0 \pm 0,4 \%)$ y eclosión $(48,6 \pm 4,2 \%)$ similares a la obtenida con DMA 8\%. DMA, a pesar de ser un crioprotector poco evaluado, ha dado buenos resultados de fertilidad y eclosión, similares a las obtenida con semen fresco, en carpa común cuando se utilizó en combinación con sucrosa (Warnecke y Pluta 2003); en Clarias gariepinus, Horváth y Urbányi (2000) registraron altas tasas de fertilidad $(83,9 \%)$ y eclosión $(61,0 \%)$ utilizando DMA 10\%; Gwo y col (1999) también observaron alta tasas de fertilidad en Onchorynchus masou ishikawae $(83,4 \pm$ $3,8 \%)$ y Onchorynchus masou formosanus $(72,1 \pm 5,7 \%)$ empleando DMA $10 \%$ y fructosa 6\%. En otras especies de peces el uso de DMA en la crioconservación de semen no ha mostrado los mejores resultados, como en el caso de Salvelinus alpinus, en concentración de $10 \%$, crioconservado en pajuelas de $2,5 \mathrm{~mL}$, se reportó fertilidad de 25,0 \pm 9,8\% (Richardson y col 2000), similar a la obtenida en el presente estudio con DMA $12 \%$.

Los resultados del presente estudio permiten sugerir que la solución crioprotectora compuesta por DMA 8\%, glucosa $6 \%$ y yema de huevo $12 \%$ es una alternativa viable para la crioconservación de semen de bocachico.

\section{REFERENCIAS}

Arroyo V, J Vergara. 2010. Efectos de la proporción ovocitossemen fresco y crioconservado en la reproducción de bocachico (Prochilodus magdalenae). Memoria de título, Programa de Acuicultura, Departamento de Ciencias
Acuícolas, Facultad de Medicina Veterinaria y Zootecnia, Universidad de Córdoba, Montería, Colombia.

Atencio V. 2001. Producción de alevinos de peces nativos. Rev MVZ Córdoba 6, 9-14.

Atencio García V, V Pertúz, E Kerguelén. 2010. Rescate de larvas de peces reofilicos en la desembocadura del rio Sinú como estrategia para diversificar las especies del programa de repoblamiento. Universidad de Córdoba/Empresa Urrá SA-ESP, Montería, Colombia.

Babiak I, J Glogowski, K Goryczko, S Dobosz, H Kuzminski, J Strzezek, W Demianowicz. 2001. Effect of extender composition and equilibration time on fertilization ability and enzymatic activity of rainbow trout cryopreserved spermatozoa. Theriogenology 56, 177-192.

Caraballo P, C Sanchéz, G Geney. 2003. La estrategia del repoblamiento un tiro al oscuro? En: Resúmenes del VII Simposio Colombiano de Icitiología, Acictios/Universidad de Córdoba, Montería, Colombia.

Carolsfeld J, HG Godinho, E Zaniboni Filho, BJ Harvey. 2003. Cryopreservation of sperm in Brazilian migratory fish conservation. J Fish Biol 63, 472-489.

CCI, Corporación Colombia Internacional. 2009. Informe de Pesca y Acuicultura Colombia 2009. CCI/MADR, Bogotá.

CCI, Corporación Colombia Internacional. 2010. Informe de Pesca y Acuicultura Colombia 2010. CCI/MADR, Bogotá.

Chao N. 1991. Fish sperm cryopreservation in Taiwan: Technology advancement and extension efforts. Academia Sinica, Monogragh. Bull Ins Zool 16, 263-283.

Ciereszko A, G Toth, S Christ, K Dabrowskila . 1996. Effect of cryopreservation and theophylline on motility characteristics of lake sturgeon (Acipenser fulvescens) spermatozoa. Theriogenology 45, 665-672.

Cóser AML, HP Godinho, D Ribeiro. 1984. Cryogenic preservation of spermatozoa from Prochilodus scrofa and Salminus maxillosus. Aquaculture 37, 387-390.

Cruz-Casallas P, V Medina-Robles, Y Velasco-Santamaría. 2006. Evaluación de diferentes crioprotectores para la crioconservación de espermatozoides de yamú (Brycon amazonicus). Rev Colomb Cienc Pecu 19, 152-159.

Dennniston R, S Michelet, KR Bondioli, RA Godke. 2011. Principles of embryo cryopreservation. In: Tiersch TH, CC Grenn (eds). Cryopreservation in aquatic species. $2^{\text {nd }}$ ed. World Aquaculture Society, Baton Rouge, Loussiana, USA, Pp 274-290.

Fogli da Silveira W, ET Kavamoto, MA Cestarolli, HM Godinho, SM Ramos, AN Silveira. 1990. Avaliação espermática, preservação criogênica e fertilidade do sêmen do pacu, Piaractus mesopotamicus (Holmberg, 1887), proveniente de reprodução induzida. $B$ Inst Pesca 17, 1-13.

Gwo C, H Ohta, K Okuzawa. 1999. Cryopreservation of sperm from the endangered formosan landlocked salmon (Oncorhyuchus masou formosanus). Theriogenology 51, 569-582.

Horváth A, B Urbányi. 2000. The effect of criyoprotectants on the motility and fertilizing capacity of cryopreserved Africant catfish (Clarias gariepinus). Aquaculture 31, 317-24.

Huanga C, Q Donga, R Walterb, T Tiersch. 2004. Initial studies on sperm cryopreservation of a live-bearing fish, the green swordtail (Xiphophorus helleri). Theriogenology 62, 179-194.

Irawan H, V Vuthiphandchai, S Nimrat. 2010. The effect of extenders, cryoprotectants and cryopreservation methods 
on common carp (Cyprinus carpio) sperm. Anim Reprod Sci 122, 236-243.

Kurland C, S Andersson. 2000. Origin and evolution of the mitochondrial proteome. Microbiol Mol Biol Rev 64, 786-820.

Lahnsteiner F, B Berger, T Weismann, R Patzner. 1996. Physiological and biochemical determination of rainbow trout, (Oncorhynchus mykiss), semen quality for cryopreservation. J Appl Aquac 6, 47-73.

Lahnsteiner F. 2011. Cryopreservation protocols for sperm of salmonid fishes. In: Tiersch TH, Grenn CC (eds). Cryopreservation in aquatic species. $2^{\text {nd }}$ ed. World Aquaculture Society, Baton Rouge, Loussiana, USA, Pp 409-420.

Li P, Q Wei, L Liu. 2008. DNA integrity of Polyodon spathula cryopreserved sperm. J Appl Ichthyol 24, 121-125.

Linhart O, M Rodina, J Cosson. 2000. Criopreservation of sperm in common carp (Cyprinus carpio) sperm motility and hatching success of embryon. Cryobiology 41, 241-250.

Maria AN, ATM Viveiros, RTF Freitas, AV Oliveira. 2006. Extenders and cryoprotectants for cooling and freezing of piracanjuba (Brycon orbignyanus) semen, an endangered Brazilian teleost fish. Aquaculture 260, 298-306.

Martínez G. 2010. Efecto del crioprotector y osmolaridad del diluyente sobre la calidad espermática y el material genético en semen crioconservado de bocachico Prochilodus magdalenae. Tesis Maestría en Biotecnología, Universidad Nacional sede Medellín, Colombia.

Martínez JG, S Pardo Carrasco. 2010. Crioconservación de semen en peces: efectos sobre la movilidad espermática y la fertilidad. Acta Biol Colomb 15, 3-24.

Martínez G, V Atencio-Garcia, S Pardo-Carrasco. 2011. Efectos de la concentración de glucosa sobre la activación de la movilidad espermática en bocachico Prochilodus magdalenae (Pisces, Characiformes). Rev MVZ Cordoba 16, 2554-2563.

McNiven MA, RK Gallant, GF Richardson. 1993. Dimethylacetamide as a cryoprotectant for rainbow trout spermatozoa. Aquaculture 40, 943-948.

Medina-Robles V, Y Velasco, P Cruz-Casallas. 2005. Aspectos generales de la crioconservación espermática en peces teleósteos. Rev Colomb Cienc Pecu 18, 34-48.

Mojica JI, C Castellanos, S Usma, R Alvarez. 2002. Libro rojo de peces dulceacuícolas de Colombia. La Serie Libros Rojos de Especies Amenazadas de Colombia. Instituto de Ciencias Naturales, Universidad Nacional de Colombia, Ministero del Medio Ambiente, Bogotá, Colombia

Moore H, M Akhondi. 1996. Fertilizing capacity of rat spermatozoa is correlated with decline in straight line speed measured by continuous computer-aided sperm analysis. J Androl 17, 50-60.

Nascimento AF, AN Maria, NO Pessoa, MAM Carvalho, ATM Viveiros. 2010. Out-of-season sperm cryopreserved in different media of the Amazonian freshwater fish pirapitinga (Piaractus brachypomus). Anim Reprod Sci 118, 324-329.

Navarro OJ, Y Velasco-Santamaría, P Cruz-Casallas. 2004. Evaluación de cinco crioprotectores para la crioconservación de semen de cachama blanca (Piaractus brachypomus). Rev Colom Cienc Pec 17, 53-59.

Ogier de Baulny B, C Labbé, G Maisse. 1999. Membrane integrity, mitochondrial activity, ATP content, and motility of the European Catfish (Silurus glanis) testicular spermatozoa after freezing with different cryoprotectans. Cryobio$\log y 39,177-184$.

Ohta H, T Izawa. 1996. Diluent for cold storage of the Japanese eel (Anguilla japonica) spermatozoa. Aquaculture 142, 107-118.

Pinzón-Arciniegas S, J Mojica-Modríguez, P Cruz-Casallas. 2005. Ensayos preliminares sobre crioconservación de bagre rayado (Pseudoplatystoma fasciatum). Orinoquia 9, 28-37.

Ramírez-Merlano J, Y Velasco-Santamaría, V Medina-Robles, P Cruz-Casallas. 2005. Crioconservación de semen de Cachama blanca (Piaractus brachypomus Cuvier, 1818): efectos del volumen de empaque y de la sustancia crioprotectora sobre la calidad seminal. Rev Colomb Cienc Pecu (resumen) 18, 331.

Ramírez-Merlano J, V Medina-Robles, P Cruz-Casallas. 2011. Crioconservación seminal de bagre rayado Pseudoplatystoma metaense (Teleostei, Pimelodidae), bajo diferentes protocolos de congelación. Arch Med Vet 43, 135-144.

Richardson G, T Miller, M McNiven. 2000. Cryopreservation of Artic charr, Salvelinus alpinus, semen in various extenders and in three sizes of Straw. Aquaculture 31, 307-15.

Rurangwa E, F Volckaert, G Huyskens, D Kime, F Ollevier. 2001. Quality control of refrigerated and cryopreserved semen using computer-assisted sperm analysis (CASA), viable staining and standardized fertilisation in African catfish (Clarias gariepinus). Theriogenology 55, 751-769.

Tiersch TR. 2011. Introduction to the second edition. In: Tiersch TH, Grenn CC (eds). Cryopreservation in aquatic species. $2^{\text {nd }}$ ed. World Aquaculture Society, Baton Rouge, Loussiana, USA, Pp 1-17.

Urbányi B, Ä Horváth, A Dinnyés. 1999. Cryopreservation of African catfish (Clarias gariepinus) sperm using different cryoprotectants. Theriogenology 51, 296.

Velasco-Santamaría YM, V Medina-Robles, P Cruz-Casallas. 2006. Cryopreservation of yamú (Brycon amazonicus) sperm for large scale fertilization. Aquaculture 256, 264-271.

Viveiros A, N So, J Komen. 2000. Sperm criopreservation of african catfish (Clarias gariepinus): Crioprotectans freezing rate and sperm: egg dilution. Theriogenology 54, 1395-1408.

Viveiros ATM, AV Oliveira, AN Maria, LH Orfão, JC Souza. 2009a. Sensibilidade dos espermatozóides de dourado (Salminus brasiliensis) a diferentes meios de congelamento. Arq Bras Med Vet Zootec 61, 883-889.

Viveiros A, L Orfaõ, A Maria, I Allaman. 2009 ${ }^{\mathrm{b}}$. A simple, inexpensive and successful freezing method forcurimba Prochilodus lineatus (Characiformes) semen. Anim Reprod Sci 112, 293-300.

Warnecke D, H Pluta. 2003. Motility and fertilizing capacity of frozen/thawed common carp (Cyprinus carpio L.) sperm using dimethyl-acetamide as the maincryoprotectant. Aquaculture 215, 165-185.

Watson P, W Holt. 2001. Cryobanking the Genetic Resource: Wildlife Conservation for the Future? Taylor and Francis, London, UK.

Wildt D, C Wemmer. 1999. Sex and wildlife: the role of reproductive science in conservation. Biodivers Conserv 8 , 965-976. 